\title{
Management of Nonalcoholic Fatty Liver Disease (NAFLD)
}

\author{
Monjur Ahmed \\ Additional information is available at the end of the chapter \\ http://dx.doi.org/10.5772/intechopen.72000
}

\begin{abstract}
Although there is an epidemic of NAFLD throughout the world, the management of NAFLD is not very satisfactory at the present time. Lifestyle modification is the main mode of therapy. Other modalities like pharmacotherapy and bariatric endoscopy or surgery should be individualized. Various pharmacological agents are being investigated to optimize the treatment of NAFLD.
\end{abstract}

Keywords: nonalcoholic fatty liver disease, fatty liver, hepatic steatosis, nonalcoholic steatohepatitis, NASH, treatment of NAFLD

\section{Introduction}

When we consider the management of Nonalcoholic Fatty Liver Disease (NAFLD), two aspects should be considered. One is that it can be a part of the metabolic syndrome [1]. About $80 \%$ of patients with metabolic syndrome have NAFLD [2]. Although the prevalence of NAFLD is $20-40 \%$ in the general population, about $70 \%$ of type 2 diabetes mellitus [3] and $85 \%$ of patients with morbid obesity (BMI $\geq 40$ ) have NAFLD [4]. In the general population, $80 \%$ of patients with NAFLD are overweight and $20 \%$ of NAFLD patients have normal weight as per ultrasonography [5]. Another aspect is that it covers a spectrum of hepatic involvement as it progresses slowly from one stage to another. Initially, it starts as simple steatosis or benign fatty liver disease or nonalcoholic fatty liver (NAFL), where there is only macrovesicular hepatic steatosis ( $>5 \%$ of hepatocytes are affected) without any inflammation, hepatocellular injury or fibrosis [6]. The second phase is nonalcoholic steatohepatitis (NASH) where there is not only hepatic steatosis but also ballooning degeneration of hepatocytes and mixed inflammatory cells (lymphocytes, plasma cells, monocytes, neutrophils and eosinophils) infiltrates mainly involving the hepatic acini [7]. The third phase is hepatic fibrosis 
which generally starts from zone 3 and progresses to bridging fibrosis, cirrhosis of liver and hepatocellular cancer. Prognosis depends on the degree of liver fibrosis [8].

\section{Purpose}

The main purpose of management of NAFLD is to halt the process as soon as it is diagnosed. The three main modalities of therapy include lifestyle modification, pharmacotherapy and bariatric surgery. Lifestyle modification is applicable to all stages of NAFLD, whereas pharmacotherapy and bariatric surgery should not be considered for patients with simple steatosis. Pharmacotherapy should be considered only for patients with biopsy-proven NASH and hepatic fibrosis as per the guideline of American Association for the Study of Liver Diseases (AASLD).

\section{Lifestyle modification}

As NAFLD is related to insulin resistance, gradual weight loss is extremely important in overweight and obese individuals [9]. Rapid weight loss can cause portal inflammation and fibrosis [10]. About $7-10 \%$ of weight loss over one year by lifestyle changes has been associated with histological improvement in simple steatosis and NASH [11]. Another study showed vigorous and moderate exercises were equally effective in reducing hepatic triglyceride content largely through weight loss [12]. Diet and moderate aerobic exercise are the first line measures to reduce weight and improve insulin resistance [13]. Dietary counseling should be highly encouraged. Consumption of high fructose containing food is the main cause of epidemic of obesity [14]. Patient should avoid high fructose containing foods like sweet, soda, desserts, breakfast cereals, granola bars and cakes. One study showed that in patients with NAFLD, fructokinase and fatty acid synthase activity are increased [15]. NAFLD may occur when there is a combination of genetic predisposition, sedentary life style and consumption of high-calorie foods [16]. One meta-analysis suggested that Omega-3 fatty acid supplementation in diet was beneficial in patients with NAFLD/NASH [17]. Patients should be encouraged to eat food rich in Omega-3 fatty acid (fish, canola, olive, perilla and chia). Food with high glycemic effects and saturated fat should be avoided [18].

In summary, lifestyle modification is the first line intervention in the management of NAFLD. This includes [1] weight loss of about $7-10 \%$ of body weight by a combination of diet and exercise [2], low-calorie diet [3], diet with high fructose and saturated fat should be avoided [4], diet with Omega-3 fatty acid supplement should be encouraged.

\section{Pharmacotherapy}

There are various pharmacological agents available for the management of NAFLD. Many of them have been found to be ineffective and some of them have high risk-benefit ratio [19]. There are various clinical trials ongoing. Here, we discuss the common agents available and the agents recommended by the American Association for the Study of Liver Diseases (ASSLD). 


\subsection{Antioxidants}

Progression of simple steatosis to steatohepatitis is related to oxidative stress and free radical formation. Vitamin $\mathrm{E}$ has been studied in different clinical trials. One study showed that patients with vitamin E deficiency and NAFLD did not respond to the classical diet for NAFLD [20]. In PIVENS trial, vitamin E 800 units per day was associated with improvement of serum transaminases and liver histology in nondiabetic NAFLD patients [21]. Fibrosis scores were not improved in this trial [22]. In SELECT trial, vitamin E supplementation 400 units per day in healthy individuals was associated with significant increase in prostate cancer [23].

Currently, vitamin E 800 units per day is recommended in nondiabetic individuals with biopsy-proven NASH [19].

\subsection{Insulin sensitizing agents}

\subsubsection{Thioglitazones (TZD)}

They are agonists/selective ligands of nuclear transcription factor PPAR- $\Upsilon$ (peroxisome proliferator-activated receptor-gamma) which is present in pancreatic $\beta$-cells, adipocytes, skeletal muscles, endothelial cells and macrophages. They increase insulin sensitivity in NAFLD and thus, promote fatty acid transportation from liver and skeletal muscles into adipose tissue, decrease serum-free fatty acid concentration and increase fatty acid oxidation in the liver [24]. Pioglitazone $30 \mathrm{mg} /$ day improved hepatic steatosis, steatohepatitis and transaminitis in nondiabetic patients with NASH in the PIVENS trial but histological response did not reach statistical significance [22]. Another study showed that in prediabetic and diabetic patients, long-term treatment with pioglitazone $45 \mathrm{mg} /$ day improved not only steatotic and inflammatory activity but also hepatic fibrosis [25]. There are few concerns about the side effects of TZD and these include weight gain [26], bone loss [27] and congestive heart failure [28].

As pioglitazone improves histology of NASH in both diabetic and nondiabetic individuals, it can be used in biopsy-proven NASH. Patients should be informed about the efficacy and side effects of this medication.

\subsubsection{Incretin-based therapy}

Glucagon-like peptide 1 (GLP-1) receptor agonists (liraglutide and exenatide) not only improves insulin sensitivity but also causes weight loss by suppressing appetite and inhibiting gastric emptying [29]. They are primarily used to control diabetes mellitus at this time. There are case reports of improvement of hepatic steatosis by GLP-1 receptor agonists [30]. Another study found that liraglutide given daily improved steatohepatitis and decreased progression of fibrosis [31].

Although incretin mimetics have been found to be helpful in diabetic patients with NAFLD, they are currently not recommended solely to treat NASH or NAFLD [19]. 


\subsubsection{Bariatric surgery}

As sustained weight loss is achievable by bariatric surgery, all the features of metabolic syndrome improve and there is reduction in mortality [32]. In a prospective study, NASH disappeared in $70 \%$ (severe NASH) to $94 \%$ (mild NASH) of patients 1 year after bariatric surgery [33]. There are various bariatric surgical and endoscopic procedures available and approved for morbid obesity at the present time. Laparoscopic sleeve gastrectomy is most commonly done in the United States [34]. Other surgical procedures include gastric bypass, biliointestinal bypass, biliopancreatic diversion with duodenal switch, vertical band gastroplasty and gastric banding. Various endoscopic procedures include intragastric balloon placement, endoscopic sleeve gastroplasty [35] and duodenal mucosal resurfacing [36]. Bariatric endoscopy is successful in reducing more weight than pharmacological agents but less effective than bariatric surgery but has less complications than bariatric surgery. Bower et al. found in a systematic review of studies that bariatric surgery improved steatosis, steatohepatitis and fibrosis in NAFLD [37]. Patients with cirrhosis of liver due to NAFLD are at a higher risk for bariatric surgery [38]. Another study showed that perioperative mortality was higher in patients with NAFLD with cirrhosis than in patients with NAFLD without cirrhosis [39].

Nowadays, bariatric surgery is not recommended as a primary treatment of NAFLD but it can be considered in obese individuals with noncirrhotic NAFLD [19].

\subsubsection{Ursodeoxycholic acid (UDCA)}

UDCA has cytoprotective effect and can improve serum transaminases in NAFLD but cannot alter liver histology [40].

UDCA is not recommended for the treatment of NAFLD or NASH [19].

\subsubsection{Omega-3 fatty acids}

Although in animal models, omega-3 fatty acid treatment improved hepatic steatosis [41, 42], recent studies did not show any significant effect on serum transaminases or liver histology [43]. Omega-3 fatty acid is not recommended for the treatment of NAFLD or NASH.

\subsubsection{Obeticholic acid (OCA)}

OCA is a ligand of farnesoid $\mathrm{X}$ receptor (FXR) which is a nuclear receptor present in liver, kidneys, intestine and adipose tissue. FXR controls target genes involved in bile acid synthesis and transport as well as lipid and carbohydrate metabolism. In the farnesoid X receptor ligand obeticholic acid in NASH treatment (FLINT) trial, OCA induced weight loss and improved hepatic fibrosis but resolution of NASH was not statistically more than placebo. OCA decreased serum transaminases but increased serum alkaline phosphatase, LDL and blood glucose levels [44].

Currently, OCA is not recommended in the routine management of NAFLD awaiting the completion of phase 3 trial (REGENERATE) of OCA for the treatment of NASH patients with liver fibrosis [45]. 


\section{Elafibranor}

Elafibranor is an agonist of PPAR- $\alpha$ and $\delta$ receptor. It has anti-inflammatory activity and can improve insulin sensitivity and lipid metabolism. It was evaluated in a phase II international study for the treatment of NASH [46]. In the post hoc analysis, elafibranor (120 mg/day for 1 year) group showed resolution of NASH without progression of fibrosis more than placebo (19\% vs. $12 \%$ ).

As the improvement was marginal, further studies are needed before using this agent in the treatment of NAFLD.

\subsection{Statins}

Hyperlipidemia is frequently seen in patients with NAFLD as part of the metabolic syndrome. Statins are commonly used for the treatment of hyperlipidemia, and low-to-moderate dose of statins have been found to be safe with low hepatic toxicity [47]. Statins decrease hepatic transaminases and hepatic fat but have no effect on hepatic fibrosis [48, 49].

Statins are not currently recommended solely for the treatment of NAFLD unless the patient has concomitant hyperlipidemia.

\subsection{Orlistat}

Orlistat is used as a weight reducing agent as it induces fat malabsorption by inhibiting enteric and pancreatic lipase [50]. A randomized controlled trial showed that orlistat improved transaminitis and hepatitis steatosis in obese individuals with NAFLD [51]. Subsequent study suggested that orlistat did not have any direct effect on NAFLD, overweight subjects improved their hepatic histology if they achieved $\geq 5 \%$ weight loss irrespective of taking orlistat [52].

Currently, orlistat cannot be recommended primarily for NAFLD.

\subsection{NAFLD and cirrhosis}

Patients should be managed the same way as in other cirrhosis. Patients with NAFLDcirrhosis have $2.6 \%$ annual cumulative risk of developing hepatocellular cancer [53]. For every 6 months, abdominal ultrasound is recommended for screening of hepatocellular carcinoma. In obese individuals, if ultrasound is technically difficult, CT or MRI should be considered. As obesity and hyperinsulinemia are risk factors for malignancy, liver cancer can occur even in noncirrhotic NAFLD [54]. Screening for esophageal and gastric varices should be done at base-line of diagnosis of cirrhosis and at regular intervals - no varices: every $2-3$ years, small varices-every 1-2 years and decompensated cirrhosis-yearly once [55].

With the epidemic of NAFLD, NASH-cirrhosis and hepatocellular carcinoma will be the leading indication of liver transplantation in future. As patients with NAFLD have multiple metabolic and cardiovascular comorbidities, they should be managed posttransplant appropriately. Management of NAFLD involves multiple specialties which include primary care physicians, gastroenterologists, hepatologists, endocrinologists, bariatric surgeons, transplant surgeons, dietitians and nutritionists. 


\section{Future therapy}

As hepatic inflammation, fibrosis, cirrhosis and subsequent malignancy are the main concerns of NAFLD, plenty of research and studies on anti-inflammatory and anti-fibrotic agents are on-going.

\section{Summary}

The management of NAFLD patients should be individualized (Table 1).

- Lifestyle change is the first line therapy: healthy food habit, increased physical activity, exercise and weight loss of $7-10 \%$.

- Pharmacotherapy is to be considered when lifestyle changes fail to achieve the goal: vitamin E in nondiabetic biopsy-proven NASH, pioglitazone in both diabetic and nondiabetic biopsyproven NASH, incretin mimetics in diabetes mellitus and NAFLD, statins in hyperlipidemia and NAFLD, orlistat in NAFLD and obesity when life-style changes fail to reduce weight loss.

- Bariatric surgery should be considered in obese individuals and noncirrhotic NAFLD.

\begin{tabular}{ll}
\hline Lifestyle changes & First line therapy of NAFLD \\
\hline Vitamin E & Nondiabetic biopsy-proven NASH \\
Pioglitazone & Both diabetic and nondiabetic biopsy-proven NASH \\
Incretin mimetics & Diabetes mellitus and NAFLD \\
Statins & Hyperlipidemia and NAFLD \\
Orlistat & NAFLD and obesity when lifestyle changes fail \\
Bariatric surgery & Morbid obesity and noncirrhotic NAFLD \\
\hline
\end{tabular}

Table 1. Summary of management of NAFLD.

\section{Author details}

Monjur Ahmed

Address all correspondence to: monjur.ahmed@jefferson.edu

Thomas Jefferson University, Philadelphia, PA, USA

\section{References}

[1] Lonardo A, Ballestri S, Marchesini G, Angulo P, Loria P. Nonalcoholic fatty liver disease: A precursor of the metabolic syndrome. Digestive and Liver Disease. 2015;47(3):181-190. DOI: 10.1016/j.dld.2014.09.020 Epub 2014 Nov 18 
[2] Antunes C, Bhimji S. Fatty Liver. StatPearls [Internet]. Treasure Island (FL): StatPearls Publishing; 2017. PMID: 28723021

[3] Leite NC, Salles GF, Araujo AL, Villela-Nogueira CA, Cardoso CR. Prevalence and associated factors of non-alcoholic fatty liver disease in patients with type-2 diabetes mellitus. Liver International. 2009;29(1):113-119. DOI: 10.1111/j.1478-3231.2008.01718.x Epub 2008 Apr 1

[4] Fabbrini E, Sullivan S, Klein S. Obesity and Nonalcoholic Fatty Liver Disease: Biochemical, Metabolic and Clinical Implications. Hepatology. 2010;51(2):679-689. DOI: 10.1002/hep.23280

[5] Bellentani S, Tiribelli C. The spectrum of liver disease in the general population: Lesson from the Dionysos study. Journal of Hepatology. 2001;35(4):531-537 PMID: 11682041

[6] Contos MJ, Sanyal AJ. The clinicopathologic spectrum and management of nonalcoholic fatty liver disease. Advances in Anatomic Pathology. 2002;9(1):37-51 PMID: 11756758

[7] Harmon RC, Tiniakos DG, Argo CK. Inflammation in nonalcoholic steatohepatitis. Expert Review of Gastroenterology and Hepatology. 2011;5(2):189-200. DOI: 10.1586/egh.11.21

[8] Angulo P, Machado MV, Diehl AM. Fibrosis in nonalcoholic Fatty liver disease: Mechanisms and clinical implications. Seminars in Liver Disease. 2015;35(2):132-145. DOI: 10.1055/ s-0035-1550065 Epub 2015 May 14

[9] Harrison SA, Day CP. Benefits of lifestyle modification in NAFLD. Gut. 2007;56(12):17601769. DOI: 10.1136/gut.2006.112094 PMCID: PMC2095707

[10] Andersen T, Gluud C, Franzmann MB, Christoffersen P. Hepatic effects of dietary weight loss in morbidly obese subjects. Journal of Hepatology. 1991;12(2):224-229 PMID: 2051001

[11] Tilg H, Moschen A. Weight loss: Cornerstone in the treatment of non-alcoholic fatty liver disease. Minerva Gastroenterologica e Dietologica. 2010;56(2):159-167

[12] Zhang HJ, He J, Pan LL, Ma ZM, Han CK, Chen CS, Chen Z, Han HW, Chen S, Sun Q, Zhang JF, Li ZB, Yang SY, Li XJ, Li XY. Effects of moderate and vigorous exercise on nonalcoholic fatty liver disease: A randomized clinical trial. JAMA Internal Medicine. 2016;176(8):1074-1082. DOI: 10.1001/jamainternmed.2016.3202

[13] Wilkins T, Tadkod A, Hepburn I, Schade RR. Nonalcoholic fatty liver disease: Diagnosis and management. American Family Physician. 2013;88(1):35-42

[14] Bray GA, Nielson SJ, Popkin BM. Consumption of high-fructose corn syrup in beverages may play a role in the epidemic of obesity. American Journal of Clinical Nutrition. 2004;79 (4):537-543

[15] Ouyang X, Cirillo P, Sautin Y, McCall S, Bruchette JL, Diehl AM, Johnson RJ, Abdelmalek MF. Fructose consumption as a risk factor for non-alcoholic fatty liver disease. Journal of Hepatology. 2008;48(6):993-999. DOI: 10.1016/j.jhep.2008.02.011 Epub 2008 Mar 10

[16] Dongiovanni P, Romeo S, Valenti L. Genetic factors in the pathogenesis of nonalcoholic fatty liver and steatohepatitis. Biomed Research International. 2015;2015:460190. DOI: 10.1155/2015/460190 Epub 2015 Jul 27. PMID: 26273621. PMCID: PMC4530215 
[17] Lu W, Li S, Li J, Wang J, Zhang R, Zhou Y, Yin Q, Zheng Y, Wang F, Xia Y, Chen K, Liu T, Lu J, Zhou Y, Guo C. Effects of omega-3 fatty acid in nonalcoholic fatty liver disease: A meta-analysis. Gastroenterology Research and Practice. 2016;2016:1459790. DOI: 10.1155/2016/1459790 PMCID: PMC5019889

[18] Ferolla SM, Silva LC, Ferrari MDLA, Cunha ASD, Martins FDS, Couto CA, Ferrari TCA. Dietary approach in the treatment of nonalcoholic fatty liver disease. World Journal of Hepatology. 2015;7(24):2522-2534. DOI: 10.4254/wjh.v7.i24.2522 PMCID: PMC4621466

[19] Chalasani N, Younossi Z, Lavine JE, Charlton M, Cusi K, Rinella M, Harrison SA, Brunt EM, Sanyal AJ. The diagnosis and management of nonalcoholic fatty liver disease: Practice guidance from the American Association for the Study of Liver Diseases. Hepatology. 2017. DOI: 10.1002/hep.29367 [Epub ahead of print]. PMID: 28714183

[20] Cankurtaran M, Kav T, Yavuz B, Shorbagi A, Halil M, Coskun T, Arslan S. Serum vitamin-E levels and its relation to clinical features in nonalcoholic fatty liver disease with elevated ALT levels. Acta Gastro-enterologica Belgica. 2006;69(1):5-11

[21] Chalasani NP, Sanyal AJ, Kowdley KV, Robuck PR, Hoofnagle J, Kleiner DE, Unalp A, Tonascia J, NASH CRN Research Group. Pioglitazone versus vitamin E versus placebo for the treatment of non-diabetic patients with non-alcoholic steatohepatitis: PIVENS trial design. Contemporary Clinical Trials. 2009;30(1):88-96. DOI: 10.1016/j.cct. 2008.09.003 Epub 2008 Sep 10

[22] Sanyal AJ, Chalasani N, Kowdley KV, McCullough A, Diehl AM, Bass NM, Neuschwander-Tetri BA, Lavine JE, Tonascia J, Unalp A, Van Natta M, Clark J, Brunt EM, Kleiner DE, Hoofnagle JH, Robuck PR, NASH CRN. Pioglitazone, vitamin E, or placebo for nonalcoholic steatohepatitis. New England Journal of Medicine. 2010;362(18):1675-1685. DOI: 10.1056/NEJMoa0907929 Epub 2010 Apr 28

[23] Klein EA, Thompson IM Jr, Tangen CM, Crowley JJ, Lucia MS, Goodman PJ, Minasian LM, Ford LG, Parnes HL, Gaziano JM, Karp DD, Lieber MM, Walther PJ, Klotz L, Parsons JK, Chin JL, Darke AK, Lippman SM, Goodman GE, Meyskens FL Jr, Baker LH. Vitamin E and the risk of prostate cancer: The Selenium and Vitamin E Cancer Prevention Trial (SELECT). Journal of the American Medical Association. 2011;306(14):1549-1556. DOI: 10.1001/ jama.2011.1437

[24] Mehta SR. Advances in the treatment of nonalcoholic fatty liver disease. Therapeutic Advances in Endocrinology and Metabolism. 2010;1(3):101-115. DOI: 10.1177/204201 8810379587 PMCID: PMC3475281

[25] Cusi K, Orsak B, Bril F, Lomonaco R, Hecht J, Ortiz-Lopez C, Tio F, Hardies J, Darland C, Musi N, Webb A, Portillo-Sanchez P. Long-term pioglitazone treatment for patients with nonalcoholic steatohepatitis and prediabetes or type 2 diabetes mellitus: A randomized trial. Annals of Internal Medicine. 2016;165(5):305-315. DOI: 10.7326/M15-1774 Epub 2016 Jun 21. PMID: 27322798

[26] Aghamohammadzadeh N, Niafar M, Dalir Abdolahinia E, et al. The effect of pioglitazone on weight, lipid profile and liver enzymes in type 2 diabetic patients. Therapeutic Advances in Endocrinology and Metabolism. 2015;6(2):56-60. DOI: 10.1177/204201881 5574229 
[27] Lecka-Czernik B. Bone loss in diabetes: Use of antidiabetic thiazolidinediones and secondary osteoporosis. Current Osteoporosis Reports. 2010;8(4):178-184. DOI: 10.1007/ s11914-010-0027 PMID: 20809203

[28] Jearath V, Vashisht R, Rustagi V, Raina S, Sharma R. Pioglitazone-induced congestive heart failure and pulmonary edema in a patient with preserved ejection fraction. Journal of Pharmacology \& Pharmacotherapeutics. 2016;7(1):41-43. DOI: 10.4103/0976-500X.179363

[29] Prasad-Reddy L, Isaacs D. A clinical review of GLP-1 receptor agonists: Efficacy and safety in diabetes and beyond. Drugs Context. 2015;4:212283. DOI: 10.7573/dic.212283 PMCID: PMC4509428

[30] Tushuizen ME, Bunck MC, Pouwels PJ, van Waesberghe JH, Diamant M, Heine RJ. Incretin mimetics as a novel therapeutic option for hepatic steatosis. Liver International. 2006;26(8):1015-1017. DOI: 10.1111/j.1478-3231.2006.01315.x 16953843

[31] Armstrong MJ, Gaunt P, Aithal GP, Barton D, Hull D, Parker R, Hazlehurst JM, Guo K, LEAN trial team, Abouda G, Aldersley MA, Stocken D, Gough SC, Tomlinson JW, Brown RM, Hübscher SG, Newsome PN. Liraglutide safety and efficacy in patients with non-alcoholicsteatohepatitis (LEAN): A multicentre, double-blind, randomised, placebo-controlled phase 2 study. Lancet. 2016;387(10019):679-690. DOI: 10.1016/S01406736(15)00803-X Epub 2015 Nov 20

[32] Adams TD, Gress RE, Smith SC, Halverson RC, Simper SC, Rosamond WD, Lamonte MJ, Stroup AM, Hunt SC. Long-term mortality after gastric bypass surgery. New England Journal of Medicine. 2007;357(8):753-761 PMID: 17715409

[33] Lassailly G, Caiazzo R, Buob D, Pigeyre M, Verkindt H, Labreuche J, Raverdy V, Leteurtre E, Dharancy S, Louvet A, Romon M, Duhamel A, Pattou F, Mathurin P. Bariatric surgery reduces features of nonalcoholic steatohepatitis in morbidly obese patients. Gastroenterology. 2015;149(2):379-388; quiz e15-6. DOI: 10.1053/j.gastro.2015.04.014 Epub 2015 Apr 25

[34] Donatelli G, Dumont JL, Cereatti F, Ferretti S, Vergeau BM, Tuszynski T, Pourcher G, Tranchart H, Mariani P, Meduri A, Catheline JM, Dagher I, Fiocca F, Marmuse JP, Meduri B. Treatment of leaks following sleeve gastrectomy by endoscopic internal drainage (EID). Obesity Surgery. 2015;25(7):1293-1301. DOI: 10.1007/s11695-015-1675-x PMID: 25913755

[35] Choi HS, Chun HJ. Recent trends in endoscopic bariatric therapies. Clinical Endoscopy. 2017;50(1):11-16. DOI: 10.5946/ce.2017.007

[36] Cherrington AD, Rajagopalan H, Maggs D, Devière J. Hydrothermal duodenal mucosal resurfacing: Role in the treatment of metabolic disease. Gastrointestinal Endoscopy Clinic of North America. 2017;27(2):299-311. DOI: 10.1016/j.giec.2016.12.002

[37] Bower G, Toma T, Harling L, Jiao LR, Efthimiou E, Darzi A, Athanasiou T, Ashrafian H. Bariatric surgery and non-alcoholic fatty liver disease: A systematic review of liver biochemistry and histology. Obesity Surgery. 2015;25(12):2280-2289. DOI: 10.1007/s11695015-1691-x 
[38] Grimm IS, Schindler W, Haluszka O. Steatohepatitis and fatal hepatic failure after biliopancreatic diversion. American Journal of Gastroenterology. 1992;87(6):775-779 PMID: 1590319

[39] Mosko JD, Nguyen GC. Increased perioperative mortality following bariatric surgery among patients with cirrhosis. Clinical Gastroenterology and Hepatology. 2011;9(10):897901. DOI: 10.1016/j.cgh.2011.07.007 Epub 2011 Jul 23

[40] Lindor KD, Kowdley KV, Heathcote EJ, Harrison ME, Jorgensen R, Angulo P, Lymp JF, Burgart L, Colin P. Ursodeoxycholic acid for treatment of nonalcoholic steatohepatitis: Results of a randomized trial. Hepatology. 2004;39(3):770-778. DOI: 10.1002/hep.20092 PMID: 14999696

[41] Di Minno MND, Russolillo A, Lupoli R, Ambrosino P, Di Minno A, Tarantino G. Omega-3 fatty acids for the treatment of non-alcoholic fatty liver disease. World Journal of Gastroenterology: WJG. 2012;18(41):5839-5847. DOI: 10.3748/wjg.v18.i41.5839

[42] ScorlettiE, Bhatia L, McCormickKG,Clough GF, NashK, Hodson L, Moyses HE, Calder PC, Byrne CD, WELCOME Study. Effects of purified eicosapentaenoic and docosahexaenoic acids in nonalcoholic fatty liver disease: Results from the Welcome* study. Hepatology. 2014;60(4):1211-1221. DOI: 10.1002/hep.27289 PMID: 25043514

[43] Sanyal AJ, Abdelmalek MF, Suzuki A, Cummings OW, Chojkier M, EPE-A Study Group. No significant effects of ethyl-eicosapentanoic acid on histologic features of nonalcoholic steatohepatitis in a phase 2 trial. Gastroenterology. 2014;147(2):377-84.e1. DOI: 10.1053/j. gastro.2014.04.046 Epub 2014 May 9

[44] Makri E, Cholongitas E, Tziomalos K. Emerging role of obeticholic acid in the management of nonalcoholic fatty liver disease. World Journal of Gastroenterology. 2016;22(41):9039-9043. DOI: 10.3748/wjg.v22.i41.9039

[45] Randomized Global Phase 3 Study to Evaluate the Impact on NASH With Fibrosis of Obeticholic Acid Treatment (REGENERATE). https://clinicaltrials.gov/ct2/show/NCT02548351

[46] Ratziu V, Harrison SA, Francque S, Bedossa P, Lehert P, Serfaty L, Romero-Gomez M, Boursier J, Abdelmalek M, Caldwell S, Drenth J, Anstee QM, Hum D, Hanf R, Roudot A, Megnien S, Staels B, Sanyal A, GOLDEN-505 Investigator Study Group. Elafibranor, an agonist of the peroxisome proliferator-activated receptor- $\alpha$ and $\delta$, induces resolution of nonalcoholic steatohepatitis without fibrosis worsening. Gastroenterology. 2016;150(5):1147-1159.e5. DOI: 10.1053/j.gastro.2016.01.038 Epub 2016 Feb 11

[47] AthyrosVG,MikhailidisDP,DidangelosTP,GioulemeOI,LiberopoulosEN,KaragiannisA, Kakafika AI, Tziomalos K, Burroughs AK, Elisaf MS. Effect of multifactorial treatment on non-alcoholic fatty liver disease in metabolic syndrome: A randomised study. Current Medical Research and Opinion. 2006;22(5):873-883

[48] Lowyck I, Fevery J. Statins in hepatobiliary diseases: Effects, indications and risks. Acta Gastro-enterologica Belgica. 2007;70(4):381-388 18330098 
[49] Eslami L, Merat S, Malekzadeh R, Nasseri-Moghaddam S, Aramin H. Statins for nonalcoholic fatty liver disease and non-alcoholic steatohepatitis. Cochrane Database of Systematic Reviews. 2013;12:CD008623. DOI: 10.1002/14651858.CD008623.pub2

[50] Guerciolini R. International Journal of Obesity and Related Metabolic Disorders: Journal of the International Association for the Study of Obesity. 1997;21(Supplement 3):S12-S23

[51] Zelber-Sagi S, Kessler A, Brazowsky E, Webb M, Lurie Y, Santo M, Leshno M, Blendis L, Halpern Z, Oren R. A double-blind randomized placebo-controlled trial of orlistat for the treatment of nonalcoholic fatty liver disease. Clinical Gastroenterology and Hepatology. 2006;4(5):639-644 Epub 2006 Apr 17

[52] Harrison SA, Fecht W, Brunt EM, Neuschwander-Tetri BA. Orlistat for overweight subjects with nonalcoholic steatohepatitis: A randomized, prospective trial. Hepatology. 2009;49(1):80-86. DOI: 10.1002/hep.22575

[53] Ascha MS, Hanouneh IA, Lopez R, Tamimi TA, Feldstein AF, Zein NN. The incidence and risk factors of hepatocellular carcinoma in patients with nonalcoholic steatohepatitis. Hepatology. 2010;51(6):1972-1978. DOI: 10.1002/hep.23527

[54] Kawada N, Imanaka K, Kawaguchi T, Tamai C, Ishihara R, Matsunaga T, Gotoh K, Yamada T, Tomita Y. Hepatocellular carcinoma arising from non-cirrhotic nonalcoholic steatohepatitis. Journal of Gastroenterology. 2009;44(12):1190-1194. DOI: 10.1007/ s00535-009-0112-0

[55] Garcia-Tsao G, Sanyal AJ, Grace ND, Carey W. Practice Guidelines Committee of the American Association for the Study of Liver Diseases; Practice Parameters Committee of the American College of Gastroenterology. Prevention and management of gastroesophageal varices and variceal hemorrhage in cirrhosis. Hepatology. 2007;46(3):922-938. DOI: 10.1002/hep.21907 PMID: 17879356 
